\title{
Wie erfolgreich ist die Katheterablation bei Vorhofflimmern?
}

\begin{abstract}
Die Übersicht zu Nutzen und Komplikationen der Katheterablation bei paroxysmalem und permanentem Vorhofflimmern stützt sich auf eine weltweite Erhebung.
\end{abstract}

- Die Datensammlung erfolgte aufgrund der Listen nationaler kardiologischer Fachgesellschaften in vier Kontinenten mit 24 Staaten bei allen Zentren, die die Katheterablation bei Vorhofflimmern anbieten. So konnten 20825 Ablationen bei 16309 Patienten (im Mittel also 1,3 Interventionen pro Patient) zwischen 2003 und 2006 in 85 Zentren ausgewertet werden. Es wurden acht verschiedene Techniken eingesetzt.

Bei einer mittleren Nachbeobachtungsdauer von zehn (mindestens vier) Monaten führte die Ablation bei etwa $80 \%$ der Patienten zu einem Sinusrhythmus. Die Erfolge sind naturgemäß bei paroxysmalem, persistierendem und permanentem Vorhofflimmern unterschiedlich und umfassen auch Patienten, die weiterhin auf Antiarrhythmika angewiesen sind. So erreicht die Erfolgsrate bei permanentem Vorhofflimmern ohne Einnahme von Antiarrhythmika nur 63\% (s. Tab. 1). Die Raten liegen bei persistierendem und permanentem Vorhofflimmern mit 61 bzw. $41 \%$ signifikant niedriger als bei der paroxysmalen Form.

Es traten $741(4,5 \%)$ schwere Komplikationen auf, darunter $25(0,15 \%)$

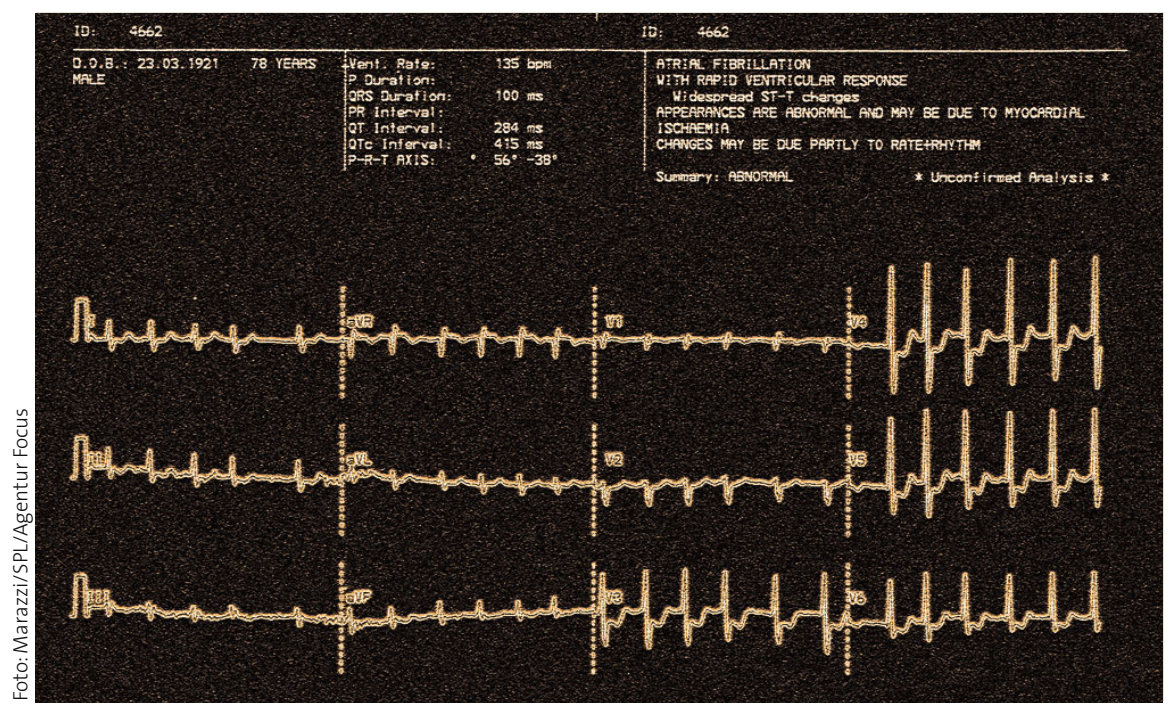

Ob Vorhofflimmern durch Katheterablation zu beheben ist, sollte mittels einer weltweiten Erhebung herausgefunden werden.

Todesfälle, $37(0,23 \%)$ Schlaganfälle, 115 (0,71\%) TIAs, 213 (1,31\%) hämorrhagische Perikardergüsse, $19(0,11 \%)$ Hämo- oder Pneumothoraxe, 216 Pulmonalstenosen über $50 \%$ (bei 48 [0,29\%] mit Intervention), $28(0,17 \%)$ permanente Zwerchfellparalysen sowie weitere 257 , teils punktionsbedingte Folgen. Außerdem kam es bei 1404 $(8,9 \%)$ Patienten zu Vorhofflattern.

\section{KOMMENTAR: Im Vergleich mit einer} ähnlichen Analyse aus den Jahren 1995 bis 2002 wurden im Zeitraum zwischen 2003 und 2006 mehr Patienten mit persistierendem und permanentem Vorhofflimmern abladiert, und es wurden auch mehr Patienten mit ungünstigen Kriterien

\section{Tabelle 1}

Erfolgsraten der Katheterablation bei verschiedenen Formen des Vorhofflimmerns (AAR = Antiarrhythmika)

\begin{tabular}{|llll|}
\hline & Ohne AAR & Mit AAR & Insgesamt \\
\hline Paroxysmal & $74,9 \%$ & $9,1 \%$ & $84,0 \%$ \\
Persistierend & $64,8 \%$ & $10,0 \%$ & $74,8 \%$ \\
Permanent & $63,1 \%$ & $7,9 \%$ & $71,0 \%$ \\
\hline
\end{tabular}

wie größeren Vorhöfen, linksventrikulärer Funktionsstörung und vorausgegangenen Herzoperationen behandelt. Deshalb sind die 10-Monats-Erfolgsraten trotz verbesserter Techniken und größerer Erfahrungen nicht angestiegen. Etwa jeder 20. Patient erleidet eine schwere Nebenwirkung. Viele Fragen bleiben unbeantwortet: Bleibt der Sinusrhythmus langfristig erhalten? Wie ist die Verbesserung der Lebensqualität bei Sinusrhythmus gegen die Notwendigkeit und Nebenwirkungen der antithrombotischen Therapie zu bewerten? Werden komplikationsfreies Überleben und Lebenserwartung verlängert?

H. HOLZGREVE

- R. Cappato et al.

Up-dated worldwide survey on the methods, efficacy and safety of catheter ablation for human atrial fibrillation. Circ. Arrhythm. Electrophysiol. http://circep.ahajournals.org 